\section{Franchi \\ T Baccetti}

\title{
Transverse maxillary deficiency in Class II and Class III malocclusions: a cephalometric and morphometric study on postero-anterior films
}

\section{Authors' affiliations:}

Lorenzo Franchi, Department of Orthodontics, The University of Florence, Florence, Italy and Thomas M. Graber Visiting Scholar, Department of Orthodontics and Pediatric Dentistry, School of Dentistry, The University of Michigan, Ann Arbor, MI, USA

Tiziano Baccetti, Department of Orthodontics, The University of Florence, Florence, Italy and Thomas M. Graber Visiting Scholar, Department of Orthodontics and Pediatric Dentistry, School of Dentistry, The University of Michigan, Ann Arbor, MI, USA

Correspondence to:

Lorenzo Franchi

Dipartimento di Odontostomatologia

Università degli Studi di Firenze

Via del Ponte di Mezzo, 46-48

50127, Firenze

Italy

Tel.: 0039055354265

Fax: 0039055609536

E-mail: 1.franchi@odonto.unifi.it

Dates:

Accepted 20 December 2004

To cite this article:

Orthod Craniofacial Res 8, 2005; 21-28

Franchi L, Baccetti T:

Transverse maxillary deficiency in Class II and Class III malocclusions: a cephalometric and morphometric study on postero-anterior films Copyright (c) Blackwell Munksgaard 2005

\section{Structured Abstract}

Authors - Franchi L, Baccetti T

Objectives - The aim of the present study is to evaluate the dentoskeletal features of subjects with either Class II or Class III malocclusions in the mixed dentition using both conventional cephalometric analysis and TPS morphometric analysis applied to posteroanterior (PA) cephalograms.

Design - TPS analyses of PA cephalograms on $49 \mathrm{Cl}-\mathrm{II}$, and 20 Cl-III subjects. Tracings were done by hand.

Setting and Sample Population - The Department of Orthodontics, University of Florence.

Outcome Measure - Size and shape differences between Cl-II and $\mathrm{Cl}-\mathrm{III}$ malocclusions.

Results - Maxillary width was smaller in both $\mathrm{Cl}-\mathrm{II}$ and $\mathrm{Cl}-\mathrm{III}$ subjects compared with normal as measured conventionally. The TPS analysis revealed transverse plane compression and extension in the vertical plane.

Conclusion - In Cl-II and $\mathrm{Cl}-\mathrm{III}$ subjects the maxillary width was smaller 2.5 and $4 \mathrm{~mm}$, respectively. TPS analyses corroborate these findings.

Key words: Class III; maxillary deficiency; transverse dimension; cephalometrics; thin-plate spline analysis

\section{Introduction}

Occlusal and craniofacial characteristics of growing subjects with either Class II or Class III molar 
relationships have been studied in the sagittal and vertical plane (1-6). Both malocclusions are complex clinical entities that entail different combinations of three-dimensional dental and skeletal components. Interestingly, studies of the transverse relationship of the maxilla to the mandible in Class II subjects in the mixed dentition have been limited to the analysis of the arch widths measured on dental casts. No information is available for dentoskeletal transverse dimensions in Class III subjects.

The transverse component of sagittal skeletal disharmonies presents clinical features that point to the need for maxillary expansion prior to correction of the anteroposterior discrepancy in growing subjects. In particular, a Class II malocclusion associated with mandibular retrusion may benefit from maxillary expansion as a first phase of therapy (7). During the post-expansion retention period, a forward posturing of the mandible can be observed in many cases, leading eventually to a spontaneous correction of the sagittal Class II relationship (8). In Class III malocclusions, rapid maxillary expansion involving protraction of the maxilla with a facemask is a common component of orthopedic treatment protocols $(7,9)$. The reasons behind this lies in the observed slight forward movement of point $\mathrm{A}$ following rapid expansion of the maxilla $(10,11)$ and in the concurrent activation of circum-maxillary sutural system during opening of the midpalatal suture (12).

\section{Transverse features of Class II malocclusion}

Fröhlich (13) compared the intercanine and intermolar widths of upper and lower arches of 51 children with Class II malocclusions with data collected by Moorrees (14) on children with normal occlusions. He found that the absolute arch widths of the children with Class II malocclusions did not differ significantly from those with normal occlusions. On the other hand, Tollaro et al. (15) have shown that an underlying negative posterior transverse interarch discrepancy (PTID; i.e. narrow maxillary arch when compared with the mandibular arch) exists in dental arches with Class II malocclusion ( $-3.4 \mathrm{~mm}$ on average) and seemingly normal buccal relationships. This underlying transverse discrepancy can be unmasked clinically by having the patient posture the mandible in an anterior position so that the canines are positioned in a Class I relationship. In 1997, Baccetti et al. (16) demonstrated that a negative
PTID is recorded consistently in Class II subjects with deciduous dentitions, and that the negative PTID is maintained or worsens during the transition into the mixed dentition. In a recent study, Varrela (17) confirmed that children with distal occlusions have narrower intermolar and intercanine distances when compared with normal subjects from the age of 3 years, and that this difference increases with age. All of these studies have been performed on dental casts, which allows for an evaluation of the width of the dental arches regardless of transverse skeletal dimensions of both jaws. To our knowledge, no data are available in the literature on the transverse dento-skeletal characteristics of Class II subjects in the mixed dentition based on the analysis of posteroanterior (PA) cephalograms.

\section{Transverse features of Class III malocclusion}

There is no definitive study of the dentoskeletal characteristics in the transverse plane of growing subjects with Class III malocclusion. The importance of the transverse dimension in Class III malocclusion is indicated indirectly by the clinical protocols of therapy, which include a preliminary phase of maxillary expansion prior to maxillary protraction $(7,9)$. In a 1995 study, Baik (18) observed significantly more favorable results of maxillary protraction in a group of Class III subjects treated with rapid maxillary expansion prior to facemask wear compared with the results in a group of Class III subjects treated only with a facemask.

\section{Morphometric approach vs. cephalometric analysis}

Conventional cephalometrics based on linear and angular measurements has shown an increasing number of limitations (19) as has the development of newer methods of biometric analyses of landmark data such as elliptic Fourier analysis; finite element analysis; tensor and shape-coordinate analysis (20-23). However, major advantages of these still evolving methods include the separate evaluation of shape (or of shape change) and of size, an optimal superimposition of landmarks for the analysis of shape change in complex skeletal configurations without the use of conventional reference lines, and an explanatory visualization of the morphological changes using transformation grids. Bookstein (24) developed morphometric approach to the comparison 
of configurations of landmarks in two or more specimens, known as thin-plate spline (TPS) analysis.

In TPS analysis, the differences in two configurations of landmarks are expressed as a continuous deformation using regression functions in which homologous points are matched between forms to minimize the bending energy (25). 'Bending energy' can be defined as the energy that would be required to bend an infinitely thin metal plate over one set of landmarks so that the height over each landmark is equal to the coordinates of the homologous point in the other form. TPS analysis enables the construction of transformation grids that capture the differences in shape and are available for visual interpretation. For a more detailed review of the theoretical base, calculation procedures, assumptions and limitations of TPS morphometrics (see 25-31). In recent times, TPS analysis has become increasingly important in orthodontics as a means of investigating modifications in shape related both to facial growth and to treatment (32-40).

The aim of the present study is to evaluate the dentoskeletal features of subjects with either Class II or Class III malocclusions in the mixed dentition using both conventional cephalometric analysis and TPS morphometric analysis applied to PA cephalograms.

\section{Subjects and methods}

Class II sample

A sample of 49 subjects ( 24 males and 25 females with a mean age of 7 years and 9 months, \pm 5 months) was selected and classified as a Class II division I malocclusion group according to the following inclusionary criteria: bilateral Class II molar relationship in centric occlusion, bilateral Class II deciduous/permanent canine relationship in centric occlusion, and buccal inclination of upper incisors.

\section{Class III sample}

A sample of 20 subjects (nine males and 11 females with a mean age of 7 years, \pm 1 year) was selected and classified as a Class III malocclusion group according to the following inclusionary criteria: bilateral Class III molar relationship in centric occlusion, bilateral Class III deciduous/permanent canine relationship in centric occlusion, and anterior crossbite.

\section{Control group}

A group of 50 subjects ( 17 boys and 33 girls with a mean age of 8 years and 4 months, \pm 3 months) with bilateral molar and deciduous/permanent canine Class I relationships in centric occlusion and no anterior or lateral cross-bites were selected as controls.

The records of all subjects were obtained from the Department of Orthodontics of the University of Florence, Italy prior to orthodontic intervention. Subjects of all groups had mixed dentitions, no missing teeth (due to aplasia, trauma, or deep caries), no history of orthodontic treatment, and did not present with craniofacial syndromes.

Posteroanterior cephalograms were of good quality for all of the subjects in all groups. All cephalograms were taken with the Frankfort plane parallel to the floor and the front of the head and the nose tip in contact with the radiographic cassette. PA cephalograms were hand-traced using $0.5 \mathrm{~mm}$ lead on $0.003 \mathrm{~mm}$ matte acetate tracing paper. All tracings were performed by one investigator and subsequently verified by another investigator. The traced PA cephalograms were analyzed using a digitizing tablet (Numonics, Landsdale, PA, USA) and Viewbox digitizing software (version 2.6). All cephalograms were enlarged $10 \%$ in order to standardize the magnification data.

To analyze the combined error of landmark location and digitization, 25 randomly selected PA cephalograms were retraced and redigitized. The standard error deviation for each dimension was calculated from double determinations using Dahlberg's formula (41). The mean value for the method error was $0.55 \pm 0.23 \mathrm{~mm}$.

\section{Conventional cephalometric analysis}

Figure 1 illustrates the bilateral cephalometric landmarks and measurements used in this part of the study.

Skeletal landmarks

Euryon (Eu): the most lateral point of the cranial vault. Medio-orbitale (Mo): the most medial point of the orbital orifice.

Latero-orbitale (Lo): the intersection of the lateral wall of the orbit and the greater wing of the sphenoid (the oblique line). 


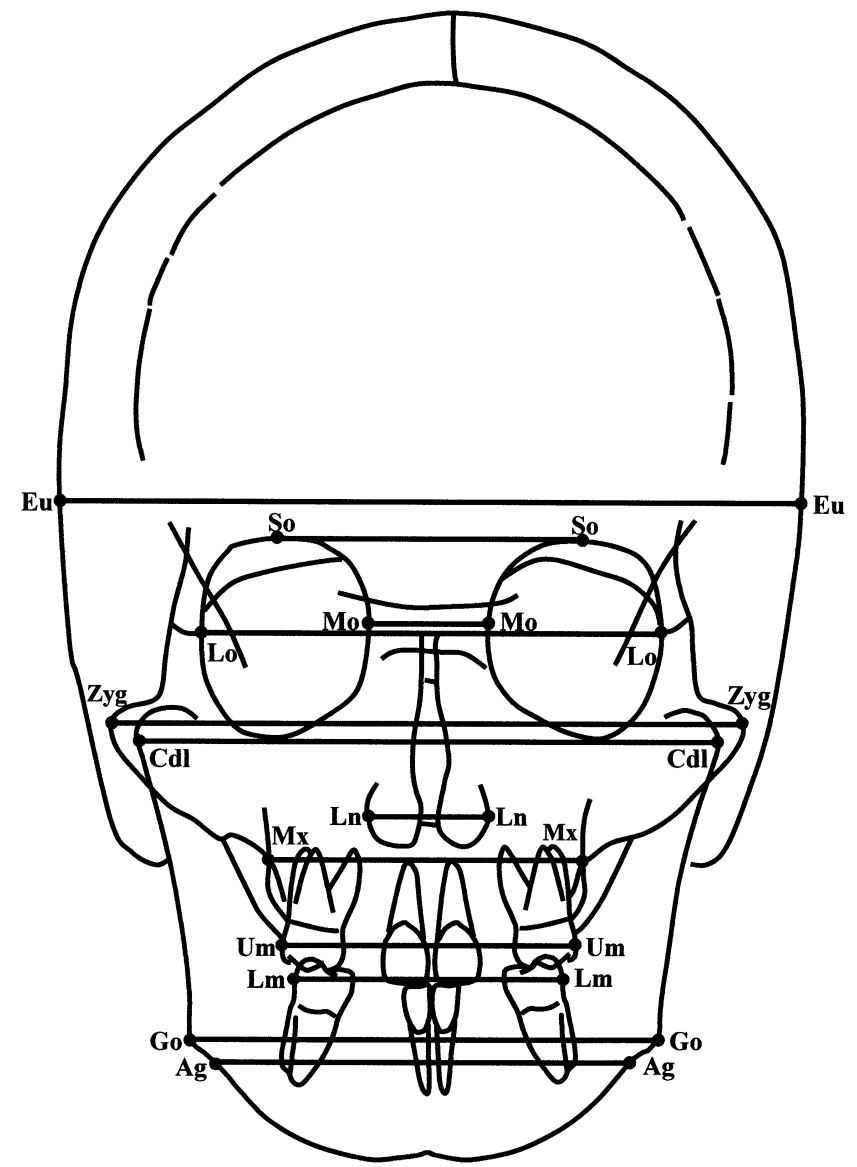

Fig. 1. Cephalometric landmarks for conventional analysis.

Supraorbitale (So): the most superior point of the orbital orifice.

Zygomatic (Zyg): the most lateral point of the zygomatic arch.

Condylar lateral ( $\mathrm{Cdl}$ ): the point located at the lateral pole of the condylar head.

Maxillare (Mx): the point located at the depth of the concavity of the lateral maxillary contour, at the junction of the maxilla and the zygomatic buttress.

Lateronasal ( $L n)$ : the most lateral point of the nasal cavity.

Gonion (Go): the point located at the gonial angle of the mandible.

Antegonion (Ag): the point located at the antegonial notch.

Dental landmarks

Upper molar (Um): the most prominent lateral point on the buccal surface of the upper first molar.
Lower molar (Lm): the most prominent lateral point on the buccal surface of the lower first molar.

From the digitized PA cephalograms, 12 width measurements (10 skeletal and two dental) were derived for each patient by connecting bilateral cephalometric landmarks.

The statistical comparisons among the three groups (Class II, Class III and controls) were performed by means of a non-parametric test (Kruskal-Wallis H test) followed by post hoc evaluation by means of the Bonferroni test; $p<0.016$ ).

\section{Thin-plate spline analysis}

In the present study, the TPS program (TpsRegr, Version 1.25; Stony Brook, University of New York, New York, USA) computed the orthogonal leastsquares Procrustes average configuration of landmarks in both the Class II group and in the control group. Following this method, every object's coordinates are translated, rotated, and scaled iteratively until the least-squared fit of all configurations is no longer improved (42). Therefore, all configurations are scaled to an equivalent size (centroid size $=1$ ) and registered with respect to one another. A description of the cephalometric landmarks used in this part of the study is illustrated in Fig. 2. Additional landmarks with respect to the conventional analysis included:

Foramen rotundum (Fr): the foramen rotundum of the sphenoid bone.

Menton (Me): the central point on the lower border of the mandibular symphisis.

Upper Interincisal Point (Ui): contact point between the upper incisors.

Lower Interincisal Point (Li): contact point between the lower incisors.

The morphometric analysis did not include point Euryon.

The average craniofacial configurations were subjected to TPS analysis to make comparisons of differences in shape between the two groups. Statistical analysis of shape differences was performed by means of permutation tests with 1000 random permutations on Wilks' Lambda statistics (42). 
Franchi and Baccetti. Transverse maxillary deficiency

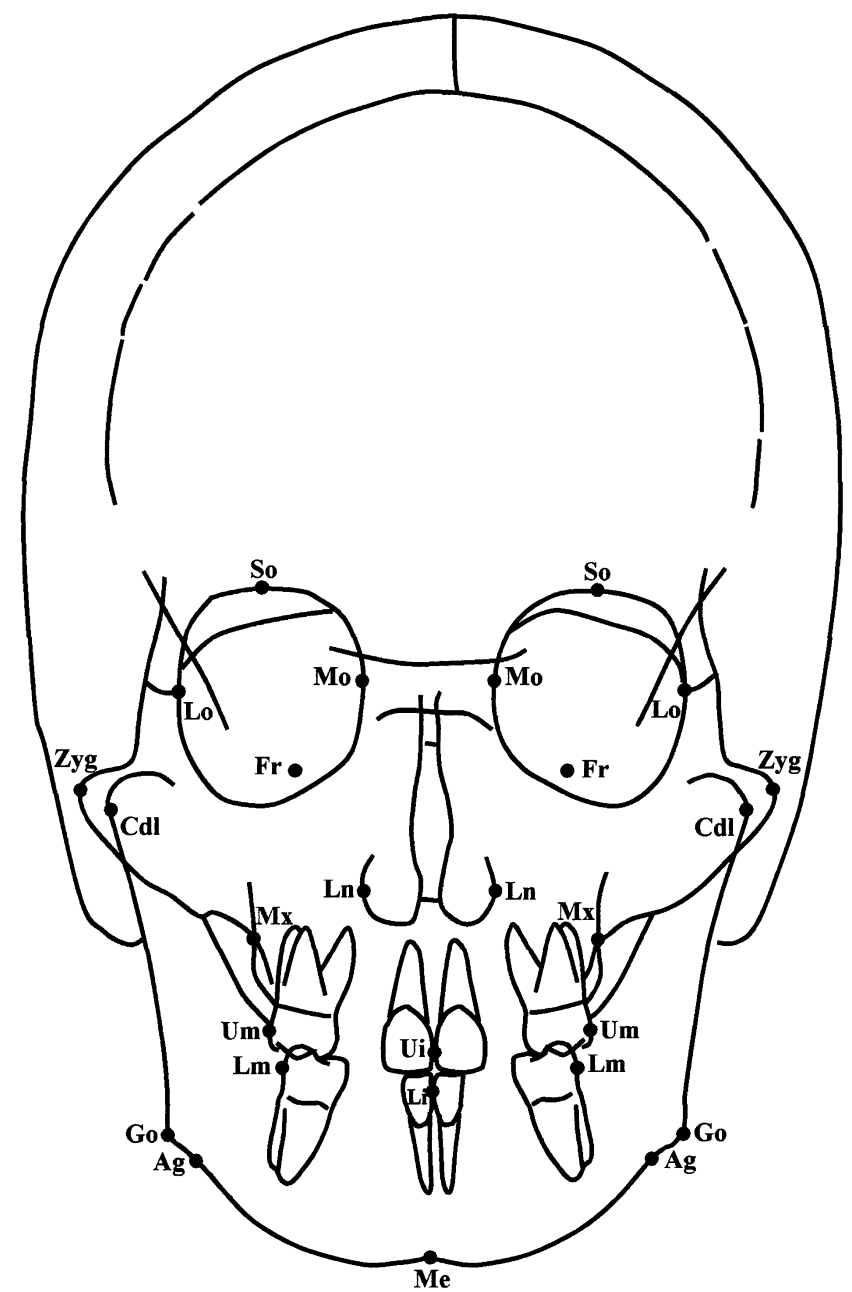

Fig. 2. Cephalometric landmarks for thin-plate spline analysis.

\section{Results}

\section{Conventional cephalometric analysis}

Maxillary width, both at the skeletal and dentoalveolar levels, was significantly smaller in both Class II and Class III groups when compared with controls. The analysis of the results showed that the measure $\mathrm{Mx}-\mathrm{Mx}$ was $2.5 \mathrm{~mm}$ smaller than normal controls in subjects with Class II malocclusion, and $3.8 \mathrm{~mm}$ smaller than normal controls in subjects with Class III malocclusion. No other significant difference was detected among the three groups for any of the remaining cephalometric measures (Table 1).

\section{Thin-plate spline analysis}

TPS analysis of PA cephalograms showed significant shape differences in the craniofacial configuration of subjects with Class II and Class III malocclusions when

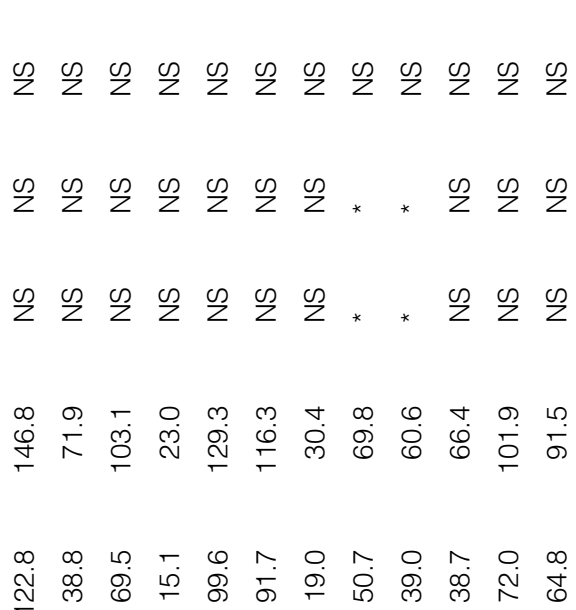


mூ



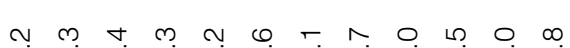



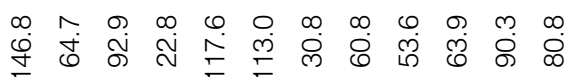



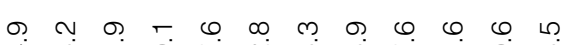
ल



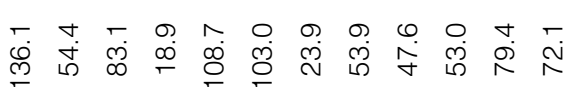

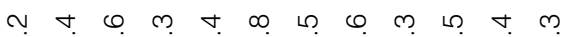
守

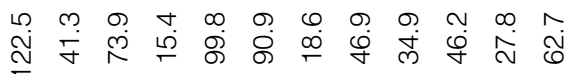

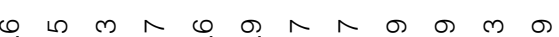
लं एँ の $\wedge$. மं


m กิ

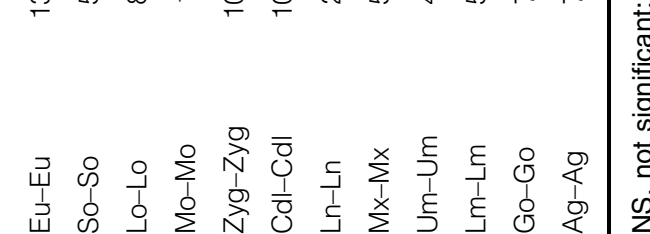


compared with control subjects $(p<0.001$; Figs 3 and 4). Analysis of the changes in transformation grids revealed very similar patterns of deformation in Class II and Class III groups. Significant shape differences occurred mainly in the maxillary region for both com-



Fig. 3. TPS graphical display of the shape differences between the Class II group and the control group (magnification factor $\times 3$ ).

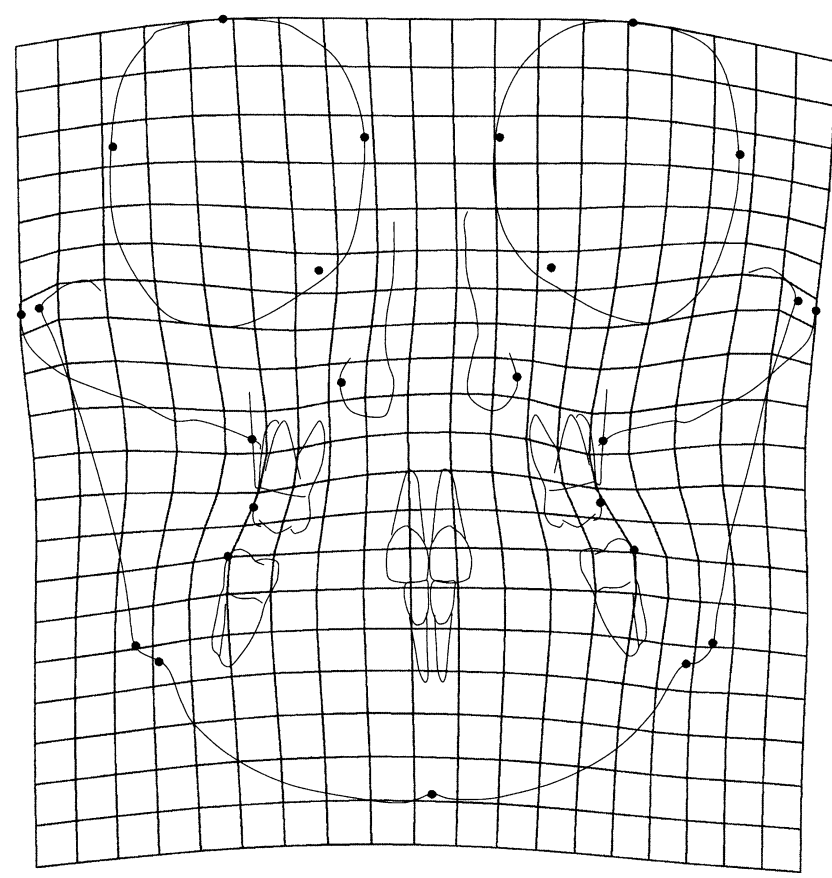

Fig. 4. TPS graphical display of the shape differences between the Class III group and the control group (magnification factor $\times 3$ ). parisons. The greatest deformation could be described as a contraction of the maxilla both at the skeletal and dental levels, i.e. a bilateral compression in the horizontal plane at point $\mathrm{Mx}$ and at point Um. This shape change in the transverse plane was most evident in the comparison between Class III group and Class I group. The contraction on the transverse plane was associated with an extension of the maxilla in the vertical plane because of a downward displacement of point $\mathrm{Mx}$ bilaterally. This vertical shape change was more accentuated in the comparison between the Class II group and the control group. No significant difference in shape could be detected in the orbital region, in the nasal region or in the mandible when comparing both Class II and Class III subjects to Class I controls, with the exception of a slight upward dislocation of point Me.

\section{Discussion}

The results of the present study show that subjects with Class II or Class III malocclusion exhibit significant size and shape differences in craniofacial configuration in the frontal plane when compared with subjects with normal occlusions. These size and shape differences mainly involved the contraction of the maxilla, both at the skeletal and dentoalveolar levels and a narrowing of the base of the nose. The reduction in skeletal width of the maxilla was associated with an increase in vertical height due to a downward displacement of point $\mathrm{Mx}$ bilaterally. No significant difference in shape was detected in the mandible on the transverse plane when comparing Class II or Class III subjects to Class I controls. The analysis of the results shows that maxillary skeletal width, on average, was $2.5 \mathrm{~mm}$ smaller in subjects with Class II malocclusions than in controls and about $4 \mathrm{~mm}$ smaller in subjects with Class III malocclusions than in Class I controls.

These findings have obvious clinical implications. As transverse deficiency in the maxilla, both at the skeletal and dentoalveolar levels, appears to be a typical feature of Class II and Class III malocclusions in the mixed dentition, an initial goal of treatment for both sagittal problems might be the early correction of the transverse occlusal relationships by means of rapid maxillary expansion (RME). Early treatment with RME is further supported by the findings of another recent 
investigation (43) that showed that patients treated before the pubertal growth spurt exhibit significant and more effective long-term changes at the skeletal level both in maxillary and circummaxillary structures. When RME treatment is performed after the pubertal peak, maxillary adaptations to expansion therapy shift from the skeletal level to the dentoalveolar level. Another study (39) that used TPS analysis to examine the long-term effects induced by RME pointed out that rapid maxillary expansion can normalize the shape of the maxillary complex in subjects presenting with transverse deficiency of the maxilla during early development. In particular, RME is able to induce transverse increments of the nasomaxillary complex that remain stable in the long-term.

In patients with mild to moderate Class II problems who have been treated during the early mixed dentition with rapid maxillary expansion followed by a palatal stabilization plate, it is not uncommon, from a clinical point of view, to observe a spontaneous correction of the Class II occlusal relationship, although no definitive Class II therapy, e.g. extraoral traction, functional jaw orthopedics, has been provided. McNamara and Brudon (7) hypothesize that expansion of the maxillary dentition may create an 'endogenous functional appliance' in that the lingual cusps of the maxillary dental arch, over expanded after RME relative to the mandibular dental arch, will encourage the growing patient to posture his or her jaw in a more protrusive position when establishing comfortable contact in centric occlusion, ultimately leading to a stable occlusal change. If spontaneous correction does not occur and for patients with a more severe skeletal and muscular problem, a functional jaw orthopedic appliance (e.g. bionator, FR-2 of Fränkel, twin block), fixed appliances with Class II elastic tractions, or headgear can be used during or slightly after the onset of the pubertal peak in growth velocity following an initial phase of expansion (44) to address the underlying anteroposterior skeletal discrepancy.

As for Class III malocclusions, the use of rapid maxillary expansion prior to facemask treatment is supported by the findings of the present study. The amount of possible post-treatment relapse in the transverse dimension suggests the overcorrection of the maxillary transverse deficiency as part of the treatment strategy in growing Class III subjects.
Acknowledgement: The authors wish to acknowledge the valuable assistance of Dr Muhieddin Alarashi in tracing and digitizing the cephalometric specimens used in this study.

\section{References}

1. Dietrich UC. Morphological variability of skeletal Class III relationships as revealed by cephalometric analysis. Trans Eur Orthod Soc 1970;131-41.

2. Moyers RE, Riolo ML, Guire KE, Wainright RL, Bookstein FL. Differential diagnosis of Class II malocclusions. Part 1. Facial types associated with Class II malocclusion. Am J Orthod 1980;78:477-94.

3. McNamara JA Jr Components of Class II malocclusion in children 8-10 years of age. Angle Orthod 1981;51:177-202.

4. Guyer EC, Ellis E, McNamara JA Jr, Behrents RG. Components of Class III malocclusion in juveniles and adolescents. Angle Orthod 1986;56:7-30.

5. Karlsen AT. Craniofacial morphology in children with Angle Class II-1 malocclusion with and without deepbite. Angle Orthod 1994;64:437-46.

6. Rothstein T, Yoon-Tarlie C. Dental and facial skeletal characteristics of males and females with Class II, Division 1 malocclusion between the ages of 10 and 14 (revisited). Part I: Characteristics of size, form and position. Am J Orthod Dentofacial Orthop 2000;117:320-32.

7. McNamara JA Jr, Brudon WL. Orthodontics and Dentofacial Orthopedics. Ann Arbor, MI: Needham Press, Inc.; 2001.

8. Wendling LK, McNamara JA Jr, Franchi L, Baccetti T. A prospective study of the short term treatment effects of the acrylicsplint rapid maxillary expander combined with the lower Schwartz appliance. Angle Orthod 2005;75:7-14.

9. Westwood PV, McNamara JAJ, Baccetti T, Franchi L, Sarver DM. Long-term effects of early Class III treatment with rapid maxillary expansion and facial mask therapy. Am J Orthod Dentofac Orthop 2003;123:306-20.

10. Haas AJ. Rapid expansion of the maxillary dental arch and nasal cavity by opening the mid-palatal suture. Angle Orthod 1961;31:73-90.

11. Dellinger EL. A preliminary study of anterior maxillary displacement. Am J Orthod 1973;63:509-16.

12. Starnbach H, Bayne D, Cleall J, Subtelny JD. Facioskeletal and dental changes resulting from rapid maxillary expansion. Angle Orthod 1966;36:152-64.

13. Fröhlich FJ. A longitudinal study of untreated Class II type malocclusion. Trans Eur Orthod Soc 1961;37:137-59.

14. Moorrees CFA. The Dentition of the Growing Child: A Longitudinal Study of Dental Development Between 3 and 18 Years of Age. Cambridge, MA: Harvard University Press; 1959.

15. Tollaro I, Baccetti T, Franchi L, Tanasescu CD. Role of posterior transverse interarch discrepancy in Class II, Division 1 malocclusion during the mixed dentition phase. Am J Orthod Dentofacial Orthop 1996;110:417-22.

16. Baccetti T, Franchi L, McNamara JA Jr, Tollaro I. Early dentofacial features of Class II malocclusion: a longitudinal study from the deciduous through the mixed dentition. Am J Orthod Dentofacial Orthop 1997;111:502-9.

17. Varrela J. Early developmental traits in Class II malocclusion. Acta Odontol Scand 1998;56:375-77. 
18. Baik HS. Clinical results of the maxillary protraction in Korean children. Am J Orthod Dentofacial Orthop 1995;108:583-92.

19. Moyers RE, Bookstein FL. The inappropriateness of conventional cephalometrics. Am J Orthod 1979;75:599-617.

20. Blum H. Biological shape and visual science. J Theor Biol 1973;38:205-87.

21. Bookstein FL. On the cephalometrics of skeletal change. Am J Orthod 1982;82:177-82.

22. Cheverud JM, Lewis JL, Bachrach W, Lew WD. The measurement of form and variation in form: an application of three-dimensional quantitative morphology by finite element methods. Am J Phys Anthropol 1983;62:151-65.

23. Lestrel PE, Roche AF. Cranial base shape variation with age: a longitudinal study of shape using Fourier analysis. Hum Biol 1986;58:527-40.

24. Bookstein FL. Principal warps: thin-plate splines and the decomposition of deformations. IEEE Trans Pattern Anal Mach Intell 1989;11:567-85.

25. Bookstein FL. Morphometric Tools for Landmark Data. New York: Cambridge University Press; 1991.

26. Bookstein FL. Biometrics, biomathematics, and the morphometric synthesis. Bull Math Biol 1996;58:313-65.

27. Bookstein FL. Shape and the information in medical images: a decade of the morphometric synthesis. Comput Vis Image Underst 1997;66:97-118.

28. Bookstein FL. A hundred years of morphometrics. Acta Zool 1998;44:7-59.

29. Rohlf FJ, Marcus LF. A revolution in morphometrics. Trend Ecol Evol 1993;8:129-32.

30. Rohlf FJ, Loy A, Corti M. Morphometric analysis of Old World Talpidae (Mammalia, Insectivora) using partial-warp scores. Syst Biol 1996;45:344-62.

31. Dryden IL, Mardia KV. Statistical Shape Analysis. New York: John Wiley \& Sons; 1998.

32. Pae EK, Lowe AA, Fleetham JA. A thin-plate spline analysis of the face and tongue in obstructive sleep apnea patients. Clin Oral Invest 1997;1:178-84.
33. Singh GD, McNamara JA Jr, Lozanoff S. Spline analysis of the mandible in human subjects with Class III malocclusion. Arch Oral Biol 1997a;42:345-53.

34. Singh GD, McNamara JA Jr, Lozanoff S. Thin-plate spline analysis of the cranial base in subjects with Class III malocclusion. Eur J Orthod 1997b;19:341-53.

35. Singh GD, McNamara JA Jr, Lozanoff S. Components of softtissue deformations in subjects with untreated Angle's Class III malocclusions: thin-plate spline analysis. J Craniofac Genet Dev Biol 1998;18:219-27.

36. Singh GD, McNamara JA Jr, Lozanoff S. Soft tissue thin-plate spline analysis of pre-pubertal Korean and European-Americans with untreated Angle's Class III malocclusions. J Craniofac Genet Dev Biol 1999;19:94-101.

37. Baccetti T, Franchi L, McNamara JA Jr. Thin-plate spline analysis of treatment effects of rapid maxillary expansion and face mask therapy in early Class III malocclusions. Eur J Orthod 1999;21:275-81.

38. Franchi L, Baccetti T, McNamara JA Jr Thin-plate spline analysis of mandibular growth. Angle Orthod 2001;71:83-9.

39. Franchi L, Baccetti T, Cameron CG, Kutcipal EA, McNamara JA Jr Thin-plate spline analysis of short-term and long-term effects of rapid maxillary expansion. Eur J Orthod 2002;24:143-50.

40. Lux CJ, Rübel J, Starke J, Conradt C, Angelika S, Komposch G. Effects of early activator treatment in patients with Class II malocclusion evaluated by thin-plate spline analysis. Angle Orthod 2001;71:120-26.

41. Dahlberg G. Statistical Methods for Medical and Biological Students. New York: Interscience Publications; 1940.

42. Rohlf FJ, Slice DE. Extensions of the Procrustes method for the optimal superimposition of landmarks. Systematic Zool 1990;39:40-59.

43. Baccetti T, Franchi L, Cameron CG, McNamara JA Jr. Treatment timing for rapid maxillary expansion. Angle Orthod 2001;71:343-50.

44. Baccetti T, Franchi L, Toth LR, McNamara JA Jr. Treatment timing for twin block therapy. Am J Orthod Dentofacial Orthop 2000;118:159-70. 